- Food and social stimuli are both effective as reinforcers for zebrafish learning.

- Response vigor is greater for food over social stimuli, but zebrafish sate rapidly.

- If food is expected, but social stimuli given, there is a negative contrast effect.

- Fish, like rats, have a cognitive representation of the goal. 


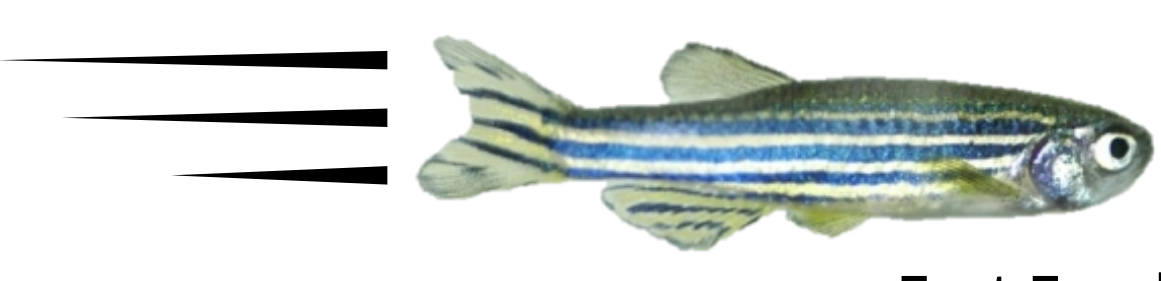

Fast Food
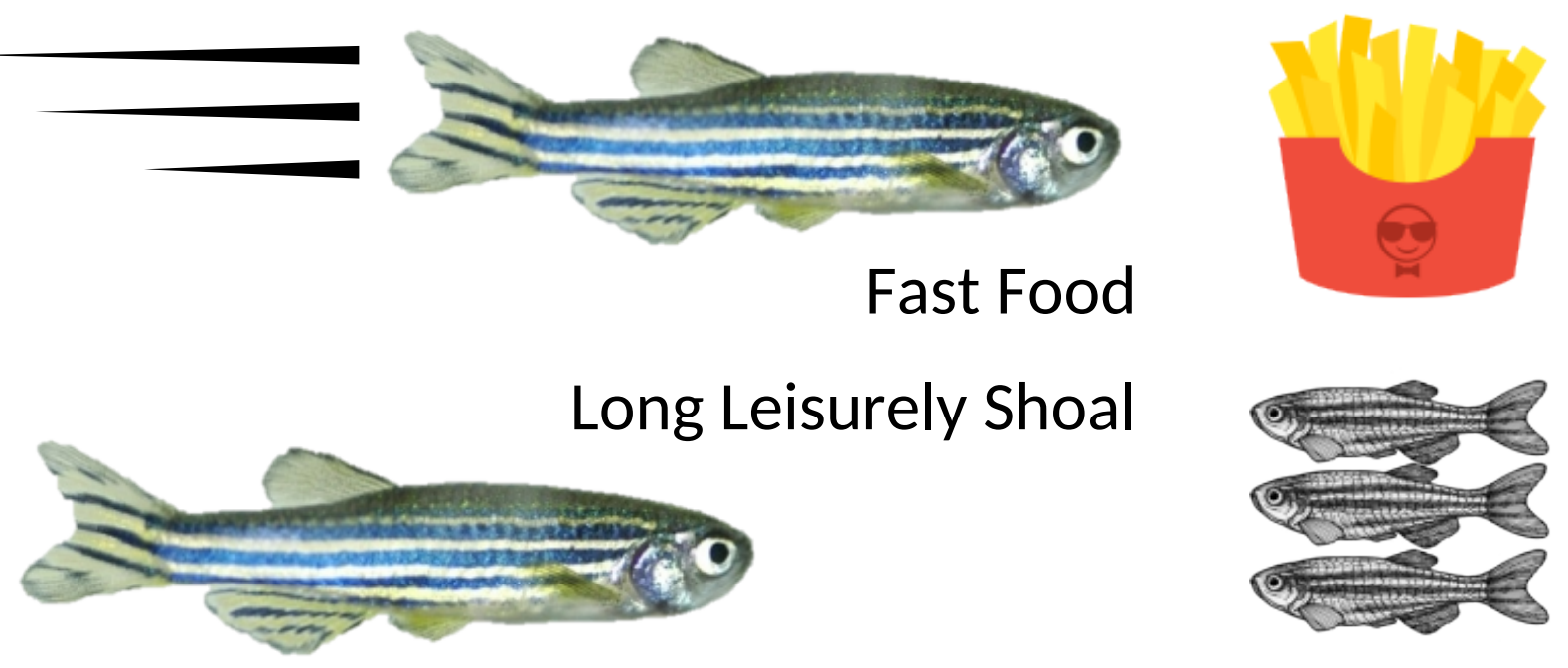


\section{Food or friends? What motivates zebrafish (Danio Rerio) performing a visual discrimination}

Jenny M. Daggett ${ }^{1,2}$, Verity J. Brown ${ }^{1}$, Caroline H. Brennan²

${ }^{1}$ School of Psychology \& Neuroscience, University of St Andrews, St Mary's Quad, South Street, St Andrews, KY16 9JP, U.K.

${ }^{2}$ School of Biological and Chemical Sciences, Queen Mary University London, Mile End Rd, E1 4NS, U.K.

Corresponding Author: Verity J Brown (https://orcid.org/0000-0001-5762-1797)

School of Psychology \& Neuroscience, University of St Andrews

St Mary's Quad, South Street, St Andrews, KY16 9JP, U.K.

vjb@st-andrews.ac.uk

Tel: +44 (0) 7792383103

Declarations of interest: none

Funding: This research did not receive any specific grant from funding agencies in the public, commercial, or not-for-profit sectors. The research was undertaken by JMD, in partial fulfillment of the requirements of a PhD degree under the joint supervision of VJB and $\mathrm{CHB}$ and supported by The University of St Andrews.

\section{Highlights}

- Food and social stimuli are both effective as reinforcers for zebrafish learning.

- Response vigor is greater for food over social stimuli, but zebrafish sate rapidly.

- If food is expected, but social stimuli given, there is a negative contrast effect.

- Fish, like rats, have a cognitive representation of the goal.

Keywords: anticipation; expectation; attention; discrimination learning; 2-AFC; fish 


\section{Abstract:}

As a model organism, zebrafish have much to offer neuroscientific research and they are increasingly being used in behavioral neuroscience, for example to study the genetics of learning and memory. As fish are often considered "less clever" than mammals, it is important to understand how they learn and to establish optimal testing conditions. In this study, we compared the efficacy of food reinforcement and social stimuli in supporting Pavlovian conditioning, Pavlovian-to-instrumental transfer, and acquisition of a twoalternative forced choice visual discrimination. Although equally effective in conditioning and in motivating discrimination learning, fish responded with shorter latencies when they were anticipating food but responded for a greater number of trials when anticipating the social stimulus. After learning, the reward was changed: food-reinforcement was replaced with the social stimulus and vice versa. Performance accuracy did not change, but response latency did: the group previously rewarded with food, but now rewarded with the social stimulus, showed a decrease in response vigor. This is a negative contrast effect, which is well established in rats, but was thought to be absent in fish because they lacked goal representation. Our results show that zebrafish, like rats, do have goal representations. Furthermore, we have shown that whereas food has greater incentive salience than social stimuli, fish become satiated rapidly, but motivation to seek social stimuli is sustained. We conclude that zebrafish are well motivated by a mixed economy of social stimuli and food. 


\section{Introduction}

The recent upsurge in the number of behavioral studies using adult zebrafish (often using tasks adapted from rodent learning research) confirms that the zebrafish is not only a suitable species for studying vertebrate cognition, but has many scientific advantages over other vertebrate species [1]. Zebrafish have been shown to be capable of performing both nonassociative and associative tasks, including: habituation [2,3]; conditioned place preference [4,5]; avoidance learning [6]; latent learning [7]; spatial reorientation [8,9]; quantity discrimination [10]; reversal learning [11]; appetitive choice discrimination [12]; and visual discrimination learning $[13,14]$.

When testing any animal, it is important that they are motivated to respond in the task. An 'incorrect' choice in a two-alternative forced choice (2-AFC) discrimination does not necessarily mean that the animal does not know the 'correct' choice: making an incorrect choice could be exploratory behavior or even a lack of interest in obtaining the reward. Therefore, one challenge in devising tasks for different species is to find species-appropriate reinforcers to support learning and ensure reliable responding. A suitable reinforcer needs to produce a high level of incentive saliency that is maintained over time and successive presentations to generate sufficient responses.

Many rodent tasks exploit natural exploratory and foraging behaviors of hungry animals, and the behavior is appetitively reinforced using food rewards. Food reward has been used successfully in zebrafish learning tasks, but with variable success, not least due to the fact that there is rapid satiation: Zebrafish can subsist without food for several days and will remain satiated for long periods after eating [15]. If the fish is not motivated to seek food, learning cannot be verified. As a shoaling species, zebrafish seek out conspecifics [16-18] and the opportunity to join a group has also been used to reinforce associative learning $[7,19,20]$. Interestingly, not only live conspecifics but also computer-simulated 'fish' are effective reinforcers [21]. One major advantage of social interaction over food reinforcement is it does not appear to satiate [19]. However, the two types of reinforcement have not previously been directly compared. Furthermore, the number of trials (i.e., reinforcer presentations) that each reinforcer will sustain in a single testing session has not been established.

Therefore, the first aim of this study was to compare the incentive salience of food and social interaction, to determine whether they would be equally effective reinforcement 
in the acquisition of a 2-AFC visual discrimination task. We were additionally interested in the impact of social interaction on the reinforcing value of food when they are presented concurrently. It is well established that zebrafish have greater foraging success when within a group and shoaling also protects against predation [22,23]. However, individuals within the group also compete for food and defend food sources from conspecifics [24,25]. Resources that are small can lead to "within-group scramble" competition, where individuals will frantically attempt to consume the food source before conspecifics can get to it [26]. Thus, feeding competition amongst hungry zebrafish might change behavior in a social context. On the other hand, concurrent reinforcement with food and social interaction might be even more effective than either reinforcer alone and support learning by maintaining motivation even while the fish become sated by the food. Finally, we aimed to establish whether zebrafish would show contrast effects $[27,28]$ if the reward was changed from food to social interaction or vice versa. The positive/negative contrast effects refer to the increase/decrease in response vigor observed when an animal is given a higher/lower value reward compared to their reward history. Although fish do show a positive contrast effect, the negative contrast effect was believed to be absent in goldfish because their behavior was determined solely by the reinforcement principle (i.e., response strength is a function of reward history and is not influenced by representation of a future reward) $[29,30]$. We therefore sought to determine whether zebrafish show contrast effects when the reward is changed.

\section{Materials and methods}

\subsection{Subjects}

A total of 30 experimentally naïve wild-type TU adult (approximately 9 months old) zebrafish (Danio rerio) of mixed sexes were obtained from the University of Edinburgh breeding facility. All fish were pair-housed in a $30 \mathrm{~L}$ tank and kept in quarantine before the beginning of the experiments for acclimatization to the laboratory environment and to monitor health. The fish were maintained in aquarium water consisting of filtered, dechlorinated water with added salts and maintained at $27-28^{\circ} \mathrm{C}$, under a constant light-dark regime, a 14:10 hr schedule (lights on at $7 \mathrm{am}$ ). The fish were fed twice daily with a diet of dry flake food supplemented with blood worm and live artemia. The exception was those being given food reward: on testing days they were not fed in the home tank and on the weekend 
days, between test days, they were fed once in the home tank. All adult behavioral testing was performed between 10:00 am and 19:00 pm, using tanks with water adjusted to the home tank temperature. Following behavioral testing, the animals were euthanized in a $500 \mathrm{mg} / \mathrm{L}$ Tricaine (Sigma-Aldrich, St. Louis, MO) buffered to $\mathrm{pH}=7.0$. All experimental procedures were authorized by UK Home Office license (Animals (Scientific Procedures) Act, 1986) and oversight of the local Animal Welfare and Ethics Committee of the University of St Andrews. This report has been prepared in accordance with the ARRIVE guidelines (https://www.nc3rs.org.uk/arrive-guidelines).

\subsection{Apparatus}

The testing apparatus was a custom-built rectangular glass aquarium (height $\mathrm{x}$ width x length: $21.5 \mathrm{~cm} \times 20.5 \mathrm{~cm} \times 29.5 \mathrm{~cm}$ ) (see Figure 1), filled to a depth of $10 \mathrm{~cm}$ with clean aquarium water. Three of the walls had non-reflective opaque coverings to block view of the outside environment. A 'holding area', $21 \mathrm{~cm}$ down the length of the tank, was created using a removable clear acrylic divider. The remaining $8.5 \mathrm{~cm}$ of the tank was divided into two 'choice areas' of equal width with an opaque barrier between them. An LCD monitor was positioned at the end wall, beyond the choice areas, for presentation of visual stimuli. The stimuli were thus presented on screen areas that were approximately $10 \mathrm{~cm}$ square and visible from the holding area.

The food reward was 2-3 ZM-400 food pellets (800 microns, 58\% protein; ZM Fish Food, UK; http://www.zmsystems.co.uk). The social reward was a 10 second animation superimposed on the colored stimulus. The animation was of 6 colored images of fish, each approximately $2 \mathrm{~cm}$ in length, moving horizontally, 3 in each direction, $21.8 \mathrm{~cm} / \mathrm{sec}$, (see Figure 2). Fish were pseudo-randomly assigned to an initial reinforcement group: social stimuli (Social); food reward (Food); or both (Social + Food) ( $n=10$ per group).

\subsection{Behavioral Procedure}

\subsubsection{Habituation}

Habituation sessions were conducted for 20 min a day over six consecutive days, to reduce stress caused from exposure to a novel environment. During each session, the experimental room lights were off, but the LCD computer monitor was projecting a grey screen, and the lights from the housing tanks in the same room provided a low level of illumination. To minimize stress from handling, netting was avoided: each fish was individually 
transported to the tank via a container filled with home tank water. The fish was allowed to swim freely in all sections of the testing tank for 20 min, after which time, the session was terminated by lowering the divider and confining the fish briefly in the holding area, before being removed from the testing tank and returned to the housing tank. For all testing sessions, there was an initial $10 \mathrm{~min}$ of habitation after the fish was introduced to the testing tank before the testing session began.

\subsubsection{Stimulus preference test}

In order to test for any preferences for either of the visual stimuli to be used in the discrimination learning, 12 fish (6 from each of the food and social groups) were given a preference test. Each fish was tested once, individually. The fish was introduced into the holding area of the testing tank and for the first 10 min the LCD monitor screen was grey. For the next $20 \mathrm{~min}$, a blue stimulus was presented in one of the choice areas and a green stimulus in the other. The position of the fish in the tank was recorded.

\subsubsection{Pavlovian conditioning and instrumental transfer}

Three test sessions were conducted on consecutive days, using the same 12 fish, to ascertain the limit in the number of rewards that the fish would work for in a single session. On the first test day, the fish underwent Pavlovian conditioning to a light stimulus. Pavlovian conditioning involves presenting a conditioned stimulus (CS) followed by an unconditioned stimulus (US). Because the CS predicts the US, it comes to elicit a conditioned response (CR). The CS was a white light, the US was either food or the animated shoal and the CR was an approach response. On the second and third test days, a Pavlovian-to-instrumental transfer test was conducted. In this procedure, the US is made contingent upon the CR: if the animal does not enter the choice areas (i.e., the $C R$ is the instrumental response) within 90 secs, the US is not delivered.

The sessions were not time limited: the session ended when the fish did not respond to the reward on three consecutive trials. For food reward, this was not consuming the reward pellet. For the social reward, this was free swimming in the tank, maintaining a distance of greater than one body length from the image of the animated shoal.

On the first two sessions, the divider was not used: the fish was allowed to swim freely throughout the testing apparatus and its position was recorded throughout. When the fish was in the holding area, a white light stimulus (CS) was projected in one of the choice areas. 
In session 1 (Pavlovian conditioning), the reward (US) was presented after 3 secs in the choice area (the food was dropped in close to the CS, or the animation was projected for 10 seconds, superimposed on the light stimulus). The light stimulus remained on for 10 seconds while the fish consumed the reward (i.e., ate the food pellet or swam within a body length of the animated shoal), after which the screen was blank for an inter-trial interval (ITI) of $10 \mathrm{sec}$.

For sessions 2 and 3, the $10 \mathrm{sec}$ ITI began when the fish returned to the holding area after swimming into one of the choice areas or if the fish failed to respond (i.e., did not enter either choice area) within $90 \mathrm{sec}$. On the third day, the divider was lowered to restrict the fish to the holding area for the duration of the ITI. After the $10 \mathrm{sec}$ ITI, the stimulus light was switched on and the divider was raised. If the fish swam into either of the choice areas, the divider was lowered, restricting the fish to that area. If the fish was in the area with the light stimulus, the reward was presented. After $10 \mathrm{sec}$, the light stimulus was terminated, and the divider was raised. When the fish swam back into the holding area, the divider was lowered, marking the end of the trial and the start of the $10 \mathrm{sec}$ ITI.

\subsubsection{Discrimination training}

Thirty fish ( $n=10$ per Reward group: Food; Social; Social + Food) began training. Eighteen fish that had not been used previously were habituated and then pre-trained in the same way as the 12 for whom data was collected (i.e., one session of Pavlovian conditioning and two sessions of Pavlovian-to-instrumental transfer; data not shown). Five of these fish failed to respond in the initial conditioning sessions and therefore the final group numbers for the 2-AFC task were: Food, $n=8$; Social, $n=9$; Social + Food, $n=8$.

For each individual (pseudo-randomly determined), one of the two visual stimuli (green or blue) was designated as 'correct'. Based on responding in the conditioning sessions, it was determined that fish would be likely to work for food for about 20 trials in a session. Therefore, a session consisted of 20 presentations of the correct and incorrect stimulus pair. For each trial, the side of the correct stimulus was pseudo-randomly determined prior to the session, with no more than 3 consecutive presentations on the same side and an equal number of trials $(n=10)$ presented to each side within a session.

After the 10 min habituation period at the start of every session, the divider was lowered confining the fish to the holding area. After a $10 \mathrm{sec}$ ITI, the stimulus pair was presented, and the divider was raised. Once the fish entered either of the choice areas, the 
divider was lowered, confining it to the choice area for $10 \mathrm{sec}$. If the fish swam into the 'correct' choice area, reinforcement was delivered immediately. There was no programmed consequence if the fish swam into the 'incorrect' choice area. After $10 \mathrm{sec}$, the stimuli were terminated, and the divider was raised to allow the fish to return to the holding area. If the fish did not spontaneously exit the choice area after $10 \mathrm{sec}$, it was gently guided back with the divider. If the fish did not enter either of the choice areas within $90 \mathrm{sec}$ of stimulus onset, the stimuli were terminated, the barrier was lowered and a $10 \mathrm{sec}$ ITI began.

After the discrimination was acquired, on Day 11 the rewards were switched, such that the Social group was now reinforced with food and the Food group was now reinforced with social stimuli. The Social + Food group continued to receive both.

The groups were compared in terms of choice accuracy and response latency (the time from stimulus onset to crossing into either choice area) over 20 days of testing.

\subsection{Data analysis}

Initial color preference testing was analyzed by calculating a bias score for individual fish to ascertain first if there were individual color preferences and then whether there was a population preference.

Conditioning (with Pavlovian-to-instrumental transfer) and discrimination learning were analyzed, separately, using repeated measures analyses of variance (ANOVA; SPSS v 24), with a between-subjects factor of Group ( 2 levels for conditioning (Social; Food); 3 levels for discrimination learning (Social; Food; Social + Food)) and a within-subject factor of Day (3 levels for conditioning; 20 levels for discrimination learning).

For conditioning, the probability of a CR (i.e., an approach) was calculated as the total number of CSs that were followed by a CR within $90 \mathrm{sec}$ as a function of the total number of CSs, excluding the final three 'no response' trials that terminated the session. For discrimination learning, accuracy was calculated as the total number of correct responses (entry to the choice area associated with reward) as a function of the number of entries into either choice areas.

In the case of significant $(p<0.05)$ interactions, simple main-effects analyses were conducted. Planned contrasts were used to confirm changes over Day and LSD tests were used to confirm the pairwise origin of Main effects of group. Where Mauchly's test of sphericity was significant, the appropriate corrected degrees of freedom were used to 
determine the critical value of $F$. Partial eta squared $\left(\eta_{p}{ }^{2}\right)$ values are reported for the estimate of effect size.

\section{Results}

\subsection{Color preference in naïve zebrafish}

Data from three sessions was lost due to equipment malfunction. Therefore, data from 9 fish were analyzed. Experimentally naïve zebrafish showed no significant color preference towards either green or blue, as indicated by an equal time spent in either choice area. There was neither a population preference nor consistent individual preference for either color. In the 20 min session, fish spent an average of $7 \mathrm{~min}$ and $43 \mathrm{sec}( \pm 27.7 \mathrm{sec})$ and $7 \mathrm{~min}$ and $32 \mathrm{sec}( \pm 24.7 \mathrm{sec})$ in the blue and green area respectively $\left(t_{(8)}=0.381, p=0.71\right)$. The fish spent $24 \%$ (range: $12-45 \%)$ of the time $(\sim 5 \mathrm{~min})$ in the larger 'holding area' of the tank and.

\subsection{Conditioning and Pavlovian-to-instrumental transfer}

The probability of an approach response within 90 secs of stimulus onset increased in all fish $(n=12)$ over the three days of conditioning, from $54 \%( \pm 0.02)$ on the first day to $74 \%$ $( \pm 0.02)$ on the third (Figure 3, right panel) (main effect of Day, $F_{(2,20)}=36.48, p<0.001, \eta_{p}{ }^{2}=$ 0.79). There was no difference between the two rewards: social stimuli or food were equally effective reinforcement overall (main effect of Reward, $F_{(1,10)}=1.55, p=0.24, \eta_{p}^{2}=0.13$ ) and the probability of an approach response increased equally rapidly in each group (Day x Group interaction, $\left.\mathrm{F}_{(2,20)}=0.65, p=0.53, \eta_{p}^{2}=0.06\right)$.

In terms of the number (as opposed to the probability) of approach responses, fish continued to respond for a greater number of trials, and so earned more than twice as many rewards, when behavior was reinforced with social stimuli compared to food (Figure 3, left panel) main effect of Group, $\left.F_{(1,10)}=32.78, p<0.001, \eta_{p}{ }^{2}=0.77\right)$. The number of rewards earned increased significantly more over the three days in the Social group, compared to the Food group (Day $x$ Group interaction, $\mathrm{F}_{(2,20)}=6.41, p=0.007, \eta_{\mathrm{p}}{ }^{2}=0.79$ ). On day three, the food-reinforcement group consumed an average of 13 food pellets (range: 12 pellets (in 14 trials) to 15 pellets (in 20 trials)), while the social-reinforcement group made an average of 37 approach responses (range: 21 approaches (in 26 trials) to 49 approaches (in 54 trials)). 


\subsection{Discrimination Performance}

Having ascertained that zebrafish do not have a bias to approach green or blue stimuli preferentially and that both social-reinforcement and food-reinforcement were equally efficacious in supporting conditioning, this experiment compared the different reinforcements, individually and together, in the acquisition and maintenance of a twoalternative forced-choice (blue-green) color discrimination. Five fish failed to respond in the initial conditioning sessions and therefore the final group numbers trained in the 2-AFC task were: Food, n=8; Social, $n=9$; Social + Food, $n=8$.

Most fish completed 20 trials in every session of the discrimination task. In particular, every fish in the Social group responded (correctly or incorrectly) on all 20 trials in every session. This is in contrast to 5/8 in the Food and and 3/8 in the Social + Food group that made omissions (i.e., failed to respond within $90 \mathrm{sec}$ of stimulus onset) (see Figure 4). The majority of omissions $(37 / 51 ; 75 \%)$ were in the first three sessions and all of these were made by four different fish in the Food group: one fish made 12 omissions on Day 1; two fish made 10 and 12 omissions on Day 2; one fish made three omissions on Day 3. In all of these cases, the omissions were the final trials of the session: on only two occasions, did these fish make an omission followed by a response. The remaining 36 omissions were runs of omissions of the final trials of the session. Another fish in the Food group made one omission, at Trial 16, on Day 17 when responding for social stimuli.

The pattern of omissions from the three fish in the Social + Food group was quite different. Overall, they made fewer of the total $(13 / 51 ; 25 \%)$ and the omissions were not in the early sessions: one fish made eight omissions on three different days (Day 7 (one); Day 9 (six); and Day 19 (one)); one fish made an omission on Day 11; and another made two omissions on Day 17. Furthermore, none of the omissions made by the Social + Food group were on the final trial and neither were more than three consecutive omissions made by these fish (see Figure 4, inset).

\subsection{Discrimination accuracy}

All groups acquired the discrimination equally rapidly, with accuracy increasing steadily over the first $\sim 10$ days of testing and maintained when the reinforcer was changed on Day 11 (main effect of Day, Greenhouse-Geisser corrected- $F_{(9.35,205.59)}=5.29, p<0.0001$, $\left.\eta_{p}{ }^{2}=0.19\right)$ (Figure 4). The improved accuracy, compared to performance on Day 1, was 
statistically reliable from Day 5 onwards (Planned contrast at Day 5: $F_{(1,22)}=5.11, p=0.03, \eta_{p}^{2}$ $=0.19$; at all subsequent days, $p<0.01, \eta_{p}{ }^{2}>0.34$ ). Accuracy was low (overall mean 65\%), but the groups were not statistically significantly different overall (main effect of Group, $F_{(2,22)}=$ 1.53, $p=0.24, \eta_{p}{ }^{2}=0.12$ ) nor in terms of rate of acquisition (Day $x$ Group interaction, $F_{(38,418)}$ $=1.04, p=0.41, \eta_{p}^{2}=0.09$ ).

\subsection{Response latency}

Latencies of correct and incorrect responses did not significantly differ (neither overall nor as a function of Day or Group: main effect and all interactions, $p<0.05$ ) therefore responses were analyzed together. Reflecting the acquisition of the discrimination, latency of responses declined over days in all groups (Figure 5; main effect of Day: Huynh-Feldt corrected $\left.-\mathrm{F}_{(3.8,132.7)}=16.01, p<0.01, \eta_{\mathrm{p}}{ }^{2}=0.42\right)$. However, notwithstanding that the different rewards supported acquisition and maintenance of responding to an equivalent degree, the nature of the reward was reflected in choice latency (interaction of Day x Group: Huynh-Feldt corrected $\left.-\mathrm{F}_{(7.6,132.7)}=2.24, p=0.03, \eta_{\mathrm{p}}{ }^{2}=0.18\right)$. The decline in latency over days was more rapid in the groups receiving food, such that Social group responded significantly more slowly than the Food group on Days 6-10 and also from the Social + Food group on Days 4, 6, 7 and 10 (Main effect of Group: Day 4: $F_{(2,22)}=3.47, p=0.05, \eta_{p}{ }^{2}=0.24$; Day 6: $F_{(2,22)}=5.13, p=0.02$, $\eta_{p}^{2}=0.32$; Day 7: $F_{(2,22)}=7.3, p=0.004, \eta_{p}^{2}=0.4$; Day 8: $F_{(2,22)}=5.3, p=0.01, \eta_{p}^{2}=0.33$; Day 9: $\mathrm{F}_{(2,22)}=5.15, p=0.02, \eta_{\mathrm{p}}{ }^{2}=0.32$; and Day 10: $\mathrm{F}_{(2,22)}=7.7, p<0.001, \eta_{\mathrm{p}}{ }^{2}=0.41$; pairwise comparisons using LSD).

The reward type was changed on Day 11 and the Social group (now receiving food) responded with the same latency as the groups who had received food to this point. However, on the fifth day after the rewards were changed, the latency of the Food group - now receiving social reward alone - was significantly slower compared to the other two groups, both receiving food. There was a significant difference between the Food and the Social and Social + Food groups on Days 15 and 17 and a difference between the Food group and the Social group (but not Social + Food group) on Days 18 and 20 (multivariate analysis, main effect of Group: Day 15: $F_{(2,22)}=4.2, p=0.03, \eta_{p}^{2}=0.28$; Day 17: $F_{(2,22)}=3.5, p=0.05, \eta_{p}^{2}=$ 0.24; Day 18: $\mathrm{F}_{(2,22)}=3.66, p=0.04, \eta_{\mathrm{p}}{ }^{2}=0.25$; and Day 20: $\mathrm{F}_{(2,22)}=4.35, p=0.03, \eta_{\mathrm{p}}{ }^{2}=0.28$, pairwise comparisons, LSD) (see Figure 5 inset)). 


\section{Discussion}

This study compared the efficacy of two different appetitive rewards - social stimuli (an animation of conspecifics) and food - to reinforce learning in zebrafish. As expected, and consistent with previous findings [11,13,14], both rewards supported Pavlovian conditioning, instrumental transfer, and acquisition of a visual 2-AFC discrimination. However, social and food rewards have different incentive salience for zebrafish and, when the rewards were exchanged, the zebrafish responded to the perceived differences in the quality of a reward, showing both positive and negative contrast effects. This challenges the assumption that fish, unlike rats, behave solely according to the reinforcement principle [29].

While both the social stimulus and the food were reinforcing, and equally efficacious in increasing the probability of approach behavior, responding in the group reinforced with the social stimulus was sustained for a significantly greater number of trials than the group that was food-reinforced. In the initial three days, fish reinforced with food alone were more likely to cease responding and not complete the 20 trials. There were no omissions by fish in the Social group, but interestingly fish reinforced with social stimuli and food did make occasional omissions, albeit uncommonly and equally likely throughout training and within the test session. This finding was not entirely unexpected: zebrafish can stay active for several days without eating and, due to their small size and low metabolic rate, they may not need to forage regularly. By contrast, seeking out conspecifics is intrinsically motivating for zebrafish and responding for social reward was sustained for longer.

All three reinforcement conditions - food, social stimuli or both together - were effective in increasing the probability of a correct choice in the 2-AFC task. The discrimination was quickly learned, with responding above chance by the fifth training day (i.e., after about 80 trials), notwithstanding that the fish in the Food group were more likely to stop responding in the initial (first three) sessions. Moreover, after the reward shift all groups maintained similar accuracy rates. We did not find evidence to suggest that using both rewards together improved response accuracy more than either of the single reward groups.

Latency to respond decreased over days but did so more dramatically in the groups anticipating food reinforcement. On Day 4 and Days 6-10, the two groups anticipating food reinforcement (with or without social reinforcement) responded to the onset of the 
discriminative stimulus with faster latencies than the fish anticipating only social reinforcement. This latency difference cannot be due to intrinsic properties of the rewards because the choice response precedes reward presentation. Therefore, the effect of reward type on latency must reflect anticipation of the expected reward and implies that anticipated food has greater incentive value to the fish than when only social reward is anticipated.

After the reward type was changed, the latency of the group that had been reinforced only with the animated shoal reduced to the same level as the previously-food-reinforced groups within the first session ( $<20$ trials), demonstrating a positive contrast effect. For the next few days of testing, the latencies of the three groups did not differ. However, on the $5^{\text {th }}$ post-shift day, the fish now receiving only the social stimuli responded with significantly reduced vigor, indicative of a negative contrast effect $[27,28]$. As goldfish were assumed not to show a negative contrast effect $[29,30]$ this requires further comment. First, it should be noted that, because we shifted both groups, we are not directly comparing the group shifted from food to social reinforcement with a group that had only ever experienced social reinforcement. Although responding at Day 15 in the now-socially-reinforced group is slower than responding at Day 10 in the same group (i.e., before the shift, when they were reinforced with food), it is not slower than - or even as slow as - the group with social reinforcement at Day 10. Nevertheless, the reduction in response latency that we report here was not found in the goldfish studied by Lowes and Bitterman (1967). We do not believe that this represents a difference between goldfish and zebrafish. Rather, procedural differences in the testing are most likely to be the reason for the apparent difference. Specifically, Lowes and Bitterman (1967) tested the goldfish for just one trial per day over 63 days, with a shift in reward magnitude at Day 23. A ten-fold increase in reward size resulted in faster response latency (a positive contrast effect) after about 20 days, which is consistent with our observation here: latency decreased within the first session (20 trials). They interpreted the positive contrast effect as unsurprising evidence that fish perceive reward size: the new, larger reward, quickly becomes part of the 'reward history' of the fish, increasing response vigor. However, they found that a 10-fold decrease in reward size did not result in slower latency (i.e., they saw no negative contrast effect) after 40 post-shift trials. They interpreted the lack of slowing in the goldfish as indicative of a difference between rats and fish. Specifically, while rats can represent goals and anticipate outcomes, they suggested that fish respond only according to 
the 'reinforcement principle', meaning that the previous, more valuable, reward would remain in the response history of the fish and so determine the response latency. However, we also did not see a slowing of latency after only 40 trials (i.e., 2 sessions), but it was evident by the $5^{\text {th }}$ session (>80 trials). This clearly demonstrates that zebrafish, like rats, do show anticipatory behavior reflecting goal representation, when they have sufficient experience of the shifted reward. It is likely that the same applies to goldfish. Although it is obvious why Lowes and Bitterman (1967) ceased testing after 40 days, this does underline the importance of ensuring that an animal is well motivated so that the experimenter can maintain control over behavior, otherwise 'unwillingness' is too readily mistaken for 'inability' to perform.

As we learn more about the behavior of zebrafish, they become an increasingly attractive model organism for use in neuroscience research (for extensive reviews see $[31,32])$. The present findings extend this knowledge by demonstrating the relative values of two different appetitive rewards. Food reward resulted in greater vigor of responding compared to anticipation of sight of an animation of conspecifics, suggesting that food has greater incentive salience. However, fish rapidly sate to food reinforcement, which limits the number of trials in each session. Using both food and the social reward together offers the benefits of both, with sustained vigorous responding. From these observations, we propose researchers use a mixed economy reinforcement regime, presenting social stimuli on a every trial ('continuous reinforcement') but presenting food reward on a Variable Ratio (VR) schedule. A VR4 schedule provides an average of 4 rewards in 10 trials, which means that this mixed economy could maintain responding for up to $\sim 50$ daily trials.

Acknowledgments: This work was supported in part by the University of St Andrews' QR block grant and the following grants awarded to CHB: NC3Rs G1000053; BBSRC BB/M007863; Leverhulme RPG-2016-143. CHB and VJB are Members of the Royal Society Industry Fellows' College.

\section{References}

[1] R. Bshary, S. Gingins, A.L. Vail, Social cognition in fishes, Trends Cogn. Sci. 18 (2014) 465-471. doi:10.1016/j.tics.2014.04.005.

[2] J.D. Best, S. Berghmans, J.J.F.G. Hunt, S.C. Clarke, A. Fleming, P. Goldsmith, A.G. Roach, Non-associative learning in larval zebrafish, Neuropsychopharmacology. 33 
(2008) 1206-1215. doi:10.1038/sj.npp.1301489.

[3] K. Wong, M. Elegante, B. Bartels, S. Elkhayat, D. Tien, S. Roy, J. Goodspeed, C. Suciu, J. Tan, C. Grimes, A. Chung, M. Rosenberg, S. Gaikwad, A. Denmark, A. Jackson, F. Kadri, K.M. Chung, A. Stewart, T. Gilder, E. Beeson, I. Zapolsky, N. Wu, J. Cachat, A. V. Kalueff, Analyzing habituation responses to novelty in zebrafish (Danio rerio), Behav. Brain Res. 208 (2010) 450-457. doi:10.1016/j.bbr.2009.12.023.

[4] P. Mathur, B. Lau, S. Guo, Conditioned place preference behavior in zebrafish, Nat. Protoc. 6 (2011) 338-345. doi:10.1038/nprot.2010.201.

[5] A.J. Brock, S.M.G. Goody, A.N. Mead, A. Sudwarts, M.O. Parker, C.H. Brennan, Assessing the Value of the Zebrafish Conditioned Place Preference Model for Predicting Human Abuse Potential, J. Pharmacol. Exp. Ther. 363 (2017) 66 LP-79. http://jpet.aspetjournals.org/content/363/1/66.abstract.

[6] X. Xu, T. Scott-Scheiern, L. Kempker, K. Simons, Active avoidance conditioning in zebrafish (Danio rerio), Neurobiol. Learn. Mem. 87 (2007) 72-77. doi:10.1016/j.nlm.2006.06.002.

[7] L.M. Gómez-Laplaza, R. Gerlai, Latent learning in zebrafish (Danio rerio), Behav. Brain Res. 208 (2010) 509-515. doi:10.1016/j.bbr.2009.12.031.

[8] S.A. Lee, G. Vallortigara, M. Flore, E.S. Spelke, V.A. Sovrano, Navigation by environmental geometry: the use of zebrafish as a model, J. Exp. Biol. 216 (2013) 3693-3699. doi:10.1242/jeb.088625.

[9] S.A. Lee, A. Ferrari, G. Vallortigara, V.A. Sovrano, Boundary primacy in spatial mapping: Evidence from zebrafish (Danio rerio), Behav. Processes. 119 (2015) 116122. doi:10.1016/j.beproc.2015.07.012.

[10] D. Potrich, V.A. Sovrano, G. Stancher, G. Vallortigara, Quantity Discrimination by Zebrafish (Danio rerio), J. Comp. Psychol. 129 (2015) 388-393. http://dx.doi.org/10.1037/com0000012.

[11] M.O. Parker, J. Gaviria, A. Haigh, M.E. Millington, V.J. Brown, F.J. Combe, C.H. Brennan, Discrimination reversal and attentional sets in zebrafish (Danio rerio), Behav. Brain Res. 232 (2012) 264-268. doi:10.1016/j.bbr.2012.04.035.

[12] J. Bilotta, M.L. Risner, E.C. Davis, S.J. Haggbloom, Assessing Appetitive Choice Discrimination Learning in Zebrafish, Zebrafish. 2 (2005) 259-268. 
doi:10.1089/zeb.2005.2.259.

[13] R.M. Colwill, M.P. Raymond, L. Ferreira, H. Escudero, Visual discrimination learning in zebrafish (Danio rerio), Behav. Processes. 70 (2005) 19-31. doi:10.1016/j.beproc.2005.03.001.

[14] K.P. Mueller, S.C.F. Neuhauss, Automated visual choice discrimination learning in zebrafish ( Danio rerio ), J. Integr. Neurosci. 11 (2012) 73-85. doi:10.1142/S0219635212500057.

[15] M. Matthews, B. Trevarrow, J. Matthews, A virtual tour of the guide for zebrafish users., Lab Anim. (NY). 31 (2002) 34-40. doi:10.1038/5000140.

[16] N. Miller, R. Gerlai, Quantification of shoaling behaviour in zebrafish (Danio rerio), Behav. Brain Res. 184 (2007) 157-166. doi:10.1016/j.bbr.2007.07.007.

[17] C. Saverino, R. Gerlai, The social zebrafish: Behavioral responses to conspecific, heterospecific, and computer animated fish, Behav. Brain Res. 191 (2008) 77-87. doi:10.1016/j.bbr.2008.03.013.

[18] R. Spence, G. Gerlach, C. Lawrence, C. Smith, The behaviour and ecology of the zebrafish, Danio rerio, Biol. Rev. 83 (2008) 13-34. doi:10.1111/j.1469185X.2007.00030.x.

[19] L. Al-Imari, R. Gerlai, Sight of conspecifics as reward in associative learning in zebrafish (Danio rerio), Behav. Brain Res. 189 (2008) 216-219. doi:10.1016/j.bbr.2007.12.007.

[20] M. Sison, R. Gerlai, Associative learning in zebrafish (Danio rerio) in the plus maze, Behav. Brain Res. 207 (2010) 99-104. doi:10.1016/j.bbr.2009.09.043.

[21] M. Qin, A. Wong, D. Seguin, R. Gerlai, Induction of Social Behavior in Zebrafish: Live Versus Computer Animated Fish as Stimuli, Zebrafish. 11 (2014) 185-197. doi:10.1089/zeb.2013.0969.

[22] C. Brown, K.N. Laland, Social learning in fishes : a review, Fish Fish. 4 (2003) 280-288. doi:10.1046/j.1467-2979.2003.00122.x.

[23] J. Krause, G.D. Ruxton, G.D. Ruxton, Living in Groups, OUP Oxford, 2002. https://books.google.com/books?id=HAoUFfVFtMcC.

[24] I.M. Hamilton, L.M. Dill, Monopolization of food by zebrafish (Danio rerio) increases in risky habitats, Can. J. Zool. 80 (2002) 2164-2169. doi:10.1139/z02-199. 
[25] M. Milinski, Games fish play: Making decisions as a social forager, Trends Ecol. Evol. 3 (1988) 325-330. doi:https://doi.org/10.1016/0169-5347(88)90088-2.

[26] B.T. Hirsch, Costs and benefits of within-group spatial position: a feeding competition model., Q. Rev. Biol. 82 (2007) 9-27. doi:10.1086/511657.

[27] L.P. Crespi, Quantitative Variation of Incentive and Performance in the White Rat, Am. J. Psychol. 55 (1942) 467. doi:10.2307/1417120.

[28] V. Di Lollo, V. Beez, Negative contrast effect as a function of magnitude of reward decrement, Psychon. Sci. 5 (1966) 99-100. doi:10.3758/BF03328300.

[29] G. Lowes, M.E. Bitterman, Reward and Learning in the Goldfish, Science. 157 (1967) 455-457.

[30] P.A. Couvillon, M.E. Bitterman, Effect of experience with a preferred food on consummatory responding for a less preferred food in goldfish, Anim. Learn. Behav. 13 (1985) 433-438. doi:10.3758/BF03208020.

[31] A. V. Kalueff, M. Gebhardt, A.M. Stewart, J.M. Cachat, M. Brimmer, J.S. Chawla, C. Craddock, E.J. Kyzar, A. Roth, S. Landsman, S. Gaikwad, K. Robinson, E. Baatrup, K. Tierney, A. Shamchuk, W. Norton, N. Miller, T. Nicolson, O. Braubach, C.P. Gilman, J. Pittman, D.B. Rosemberg, R. Gerlai, D. Echevarria, E. Lamb, S.C.F. Neuhauss, W. Weng, L. Bally-Cuif, H. Schneider, and the Zebrafish Neuros, Towards a Comprehensive Catalog of Zebrafish Behavior 1.0 and Beyond, Zebrafish. 10 (2013) 70-86. doi:10.1089/zeb.2012.0861.

[32] A.M. Stewart, A. V. Kalueff, Developing better and more valid animal models of brain disorders, Behav. Brain Res. 276 (2015) 28-31. doi:10.1016/j.bbr.2013.12.024. 


\section{Figure Legends}

Figure 1. Schematic of the testing tank. The sides of the glass tank were covered with a custom-built acrylic mask to occlude the view of the room and so avoid distraction. A transparent removable barrier divided the larger holding area from two choice areas. The tank was filled to a depth of $10 \mathrm{~cm}$ with aquarium water. The tank was positioned so that stimuli could be seen on an LCD computer monitor at one end of the tank. The viewing area was approximately $10 \mathrm{~cm}$ square on each side of an opaque central divider. A camera mounted above and lines on the floor of the tank enabled accurate judgement of the position of the fish.

Figure 2: Still image of the social reward stimulus: arrows show the direction of movement of each 'fish' when the image was animated. The six 'fish' were colored and approximately $2 \mathrm{~cm}$ in length. Each image moved horizontally (three from left to right and three from right to left) at a constant speed of $21.8 \mathrm{~cm} / \mathrm{sec}$. across the approximately-10cm square stimulus viewing area, reappearing on the other side as they moved out of the viewing area in a constant loop. The experimental animal was deemed to have responded to the animation if it shoaled with the animated fish (i.e., moved at speed in either direction, within one body length of the screen). The six 'fish' were presented with no background, or indeed any other stimulus elements (the arrows and speed information have been added to this schematic). The animated fish were superimposed on the colored stimulus to which a correct response had been made.

Figure 3. Left Panels: Mean ( \pm SEM, but which are occluded by the symbols for the foodreinforced group) number of conditioned responses (CRs) to the conditioned stimulus (CS). The session ended after 3 consecutive presentations of the CS did not elicit a CR. Day 1 was Pavlovian Conditioning (the food or social stimulus (i.e., the unconditioned stimulus, US) was contingent only on the CS). Days 2 and 3, were Pavlovian-to-instrumental-transfer, where the US was contingent on the fish making the CR. Both groups showed more CRs as a function of Day, but fish in the Food group showed a smaller increase over Days and earned fewer rewards than the fish in the Social group on all three Days $\left({ }^{*} p<.05\right)$. Right Panels: Excluding the three trials that terminated the session, the likelihood of a CR following a CS 
was equivalent for the two groups, indicating that the food and social stimuli were equally salient and reinforcing as USs, but that the response to food sated more rapidly.

Figure 4. Total number of omissions in the 2-AFC discrimination made by the Food and Social + Food groups (the Social group made no omissions). In the Food group, four fish together made a total 37 omissions in the first three Days, representing $75 \%$ of all omissions in the experiment. There were no omissions on these Days by fish in the Social + Food group. The inset graph shows omissions by trial number, collapsed over days. The omissions in the Food group were made at the end of the sessions; the omissions in the Social + Food group were distributed throughout the session.

Figure 5. Mean ( \pm SEM) of accuracy for the 2-AFC discrimination. Accuracy increased over days for all three groups. The dashed line indicates chance level responding (50\%) and the vertical arrow and line break indicates the point of the reward shift. On Day 5 and all subsequent days, accuracy was significantly greater than on Day $1\left({ }^{*} p<0.05\right)$.

Figure 6. Mean ( \pm SEM) latency of responses for the 2-AFC discrimination. Latency decreased over days for all three groups, but the decrease was greater for the two groups reinforced with food compared to the Social group. The Social group responded significantly more slowly compared Food group on Days 6-10 (* $p<0.05)$ and from the Social + Food group on Days 4, 6, 7 and $10(+p<0.05)$. After the reward shift, the Social group showed a positive contrast effect and sped up to the level of the other two groups. The inset graph blows up the $y$-axis to enable appreciation of the new group difference emerging at Day 15 ( $5^{\text {th }}$ day post-shift): the group now receiving the social stimulus is significantly slower than both of the other two groups on Days 15 and $17(+p<0.05)$. and slower than the Social group (now receiving food) on Days 15, 17, 18 and $20\left({ }^{*} p<0.05\right)$. 


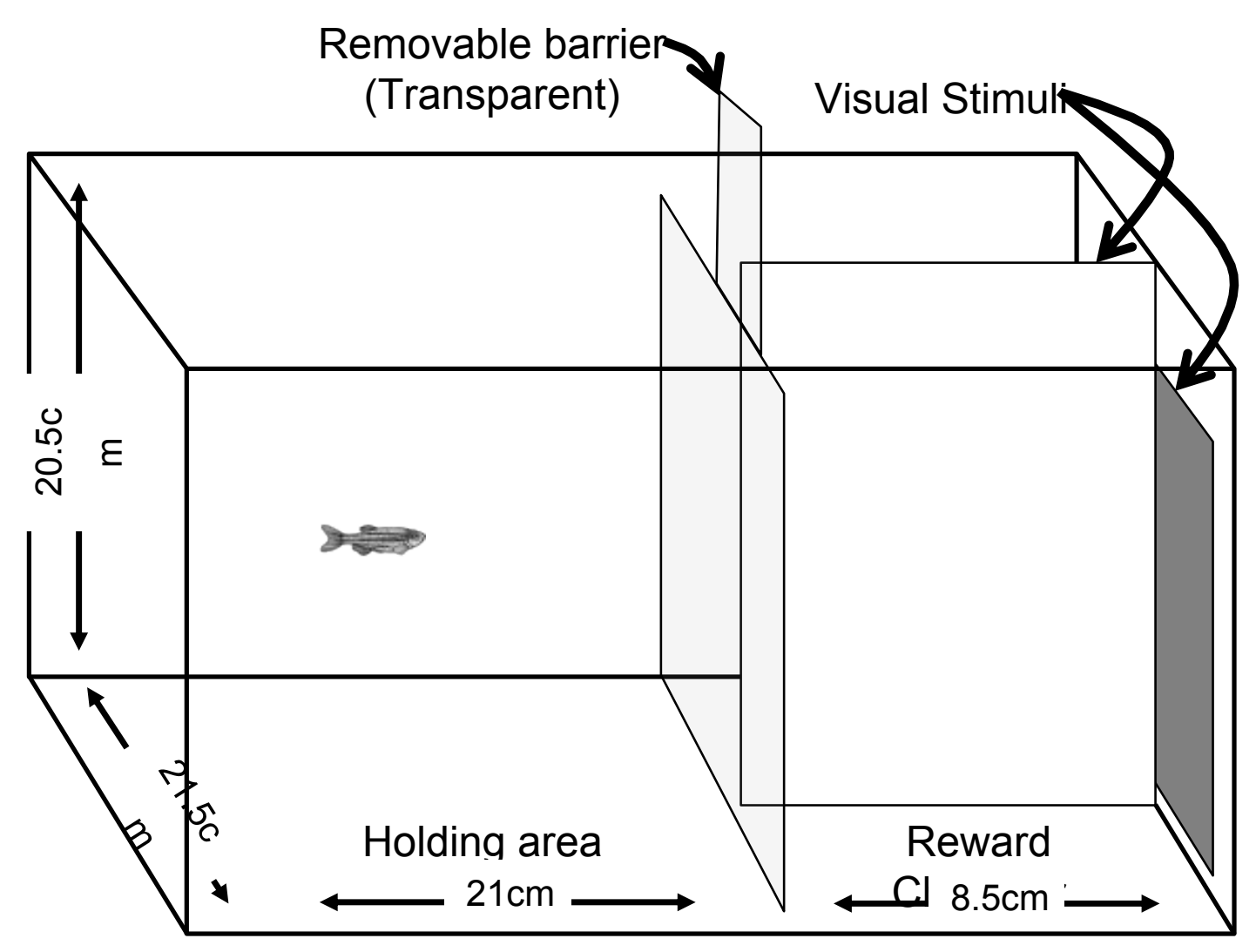




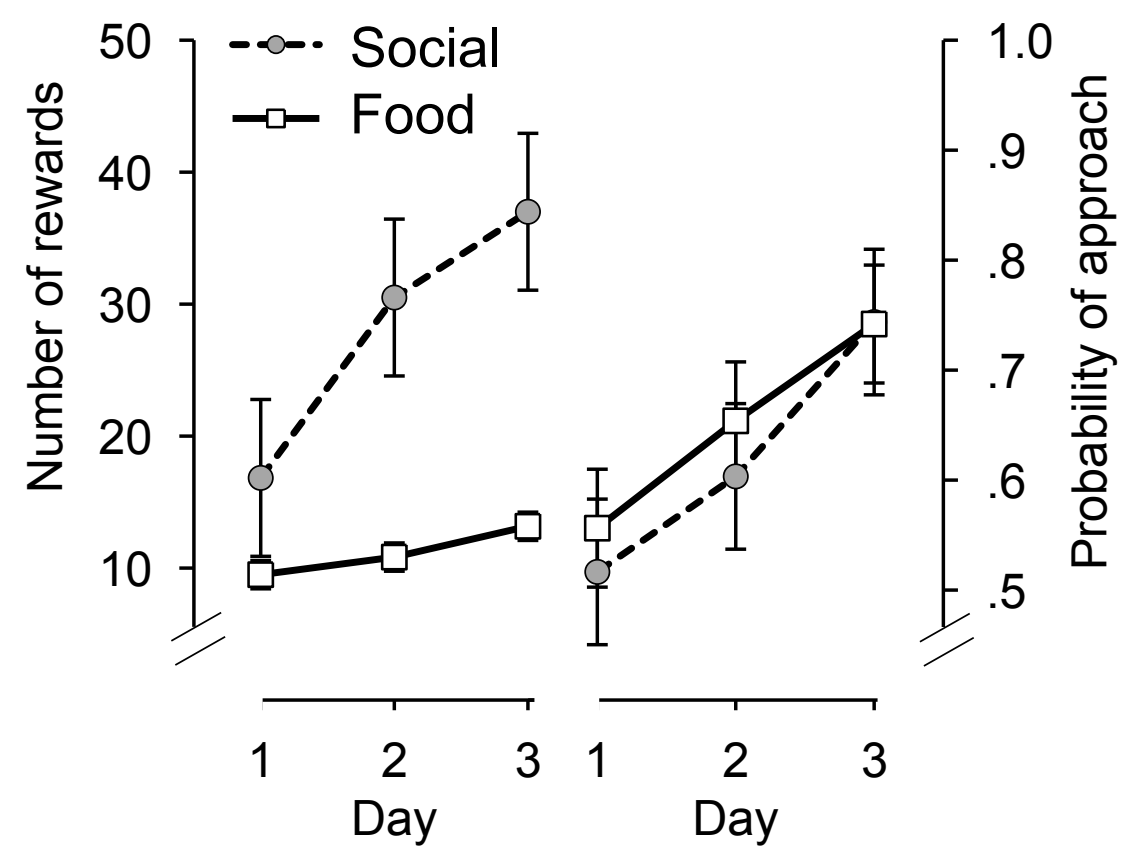




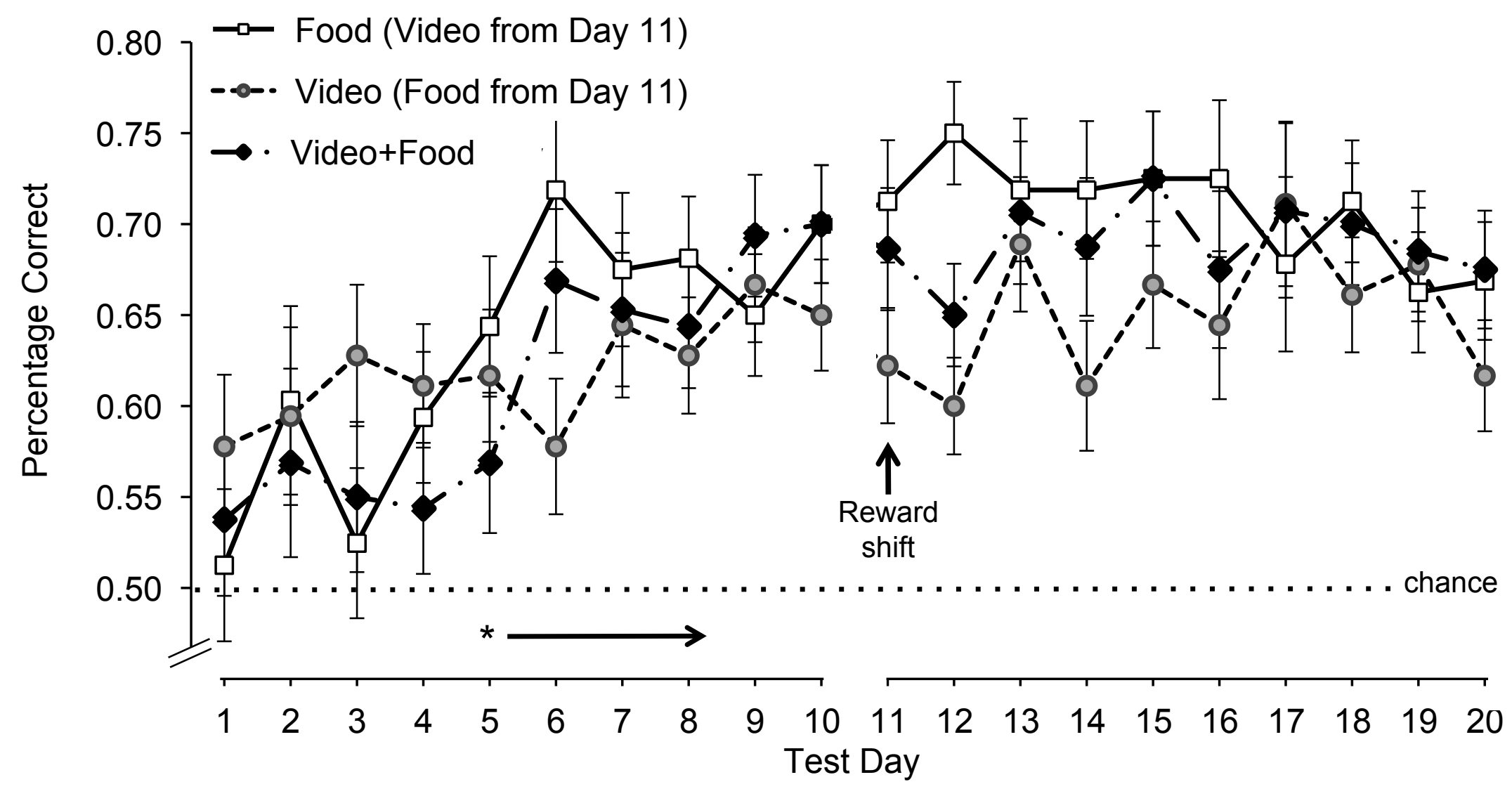




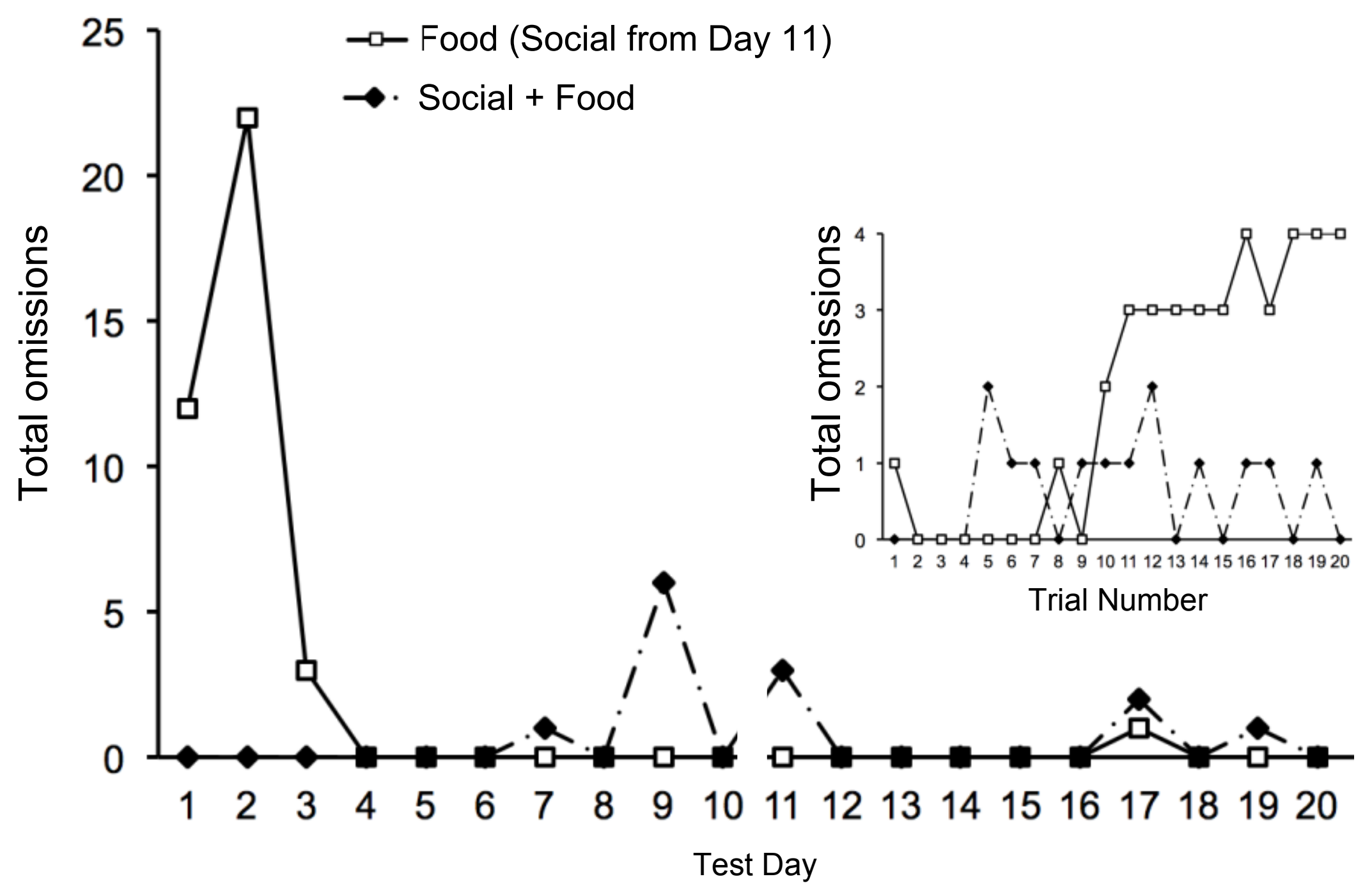




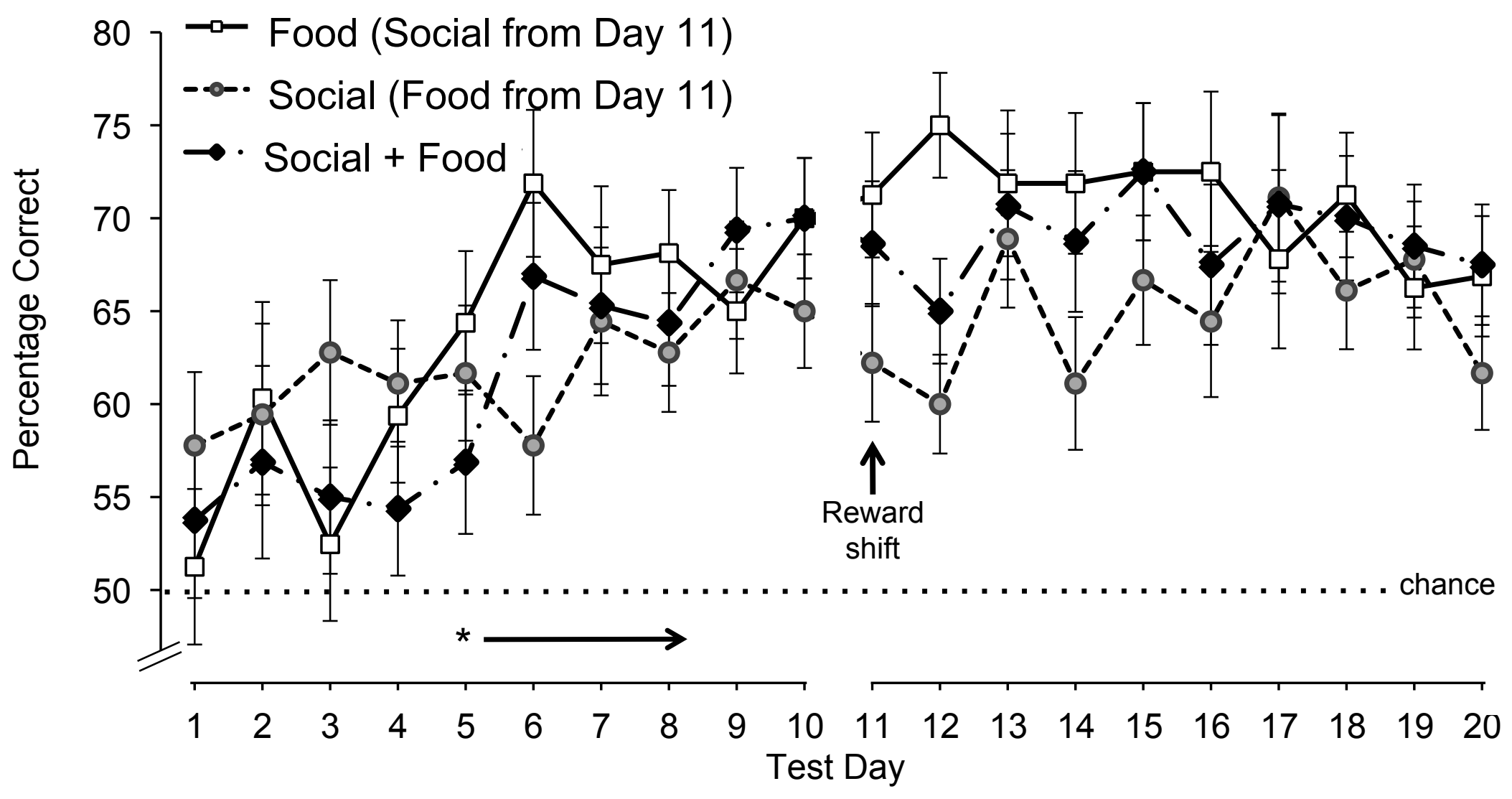




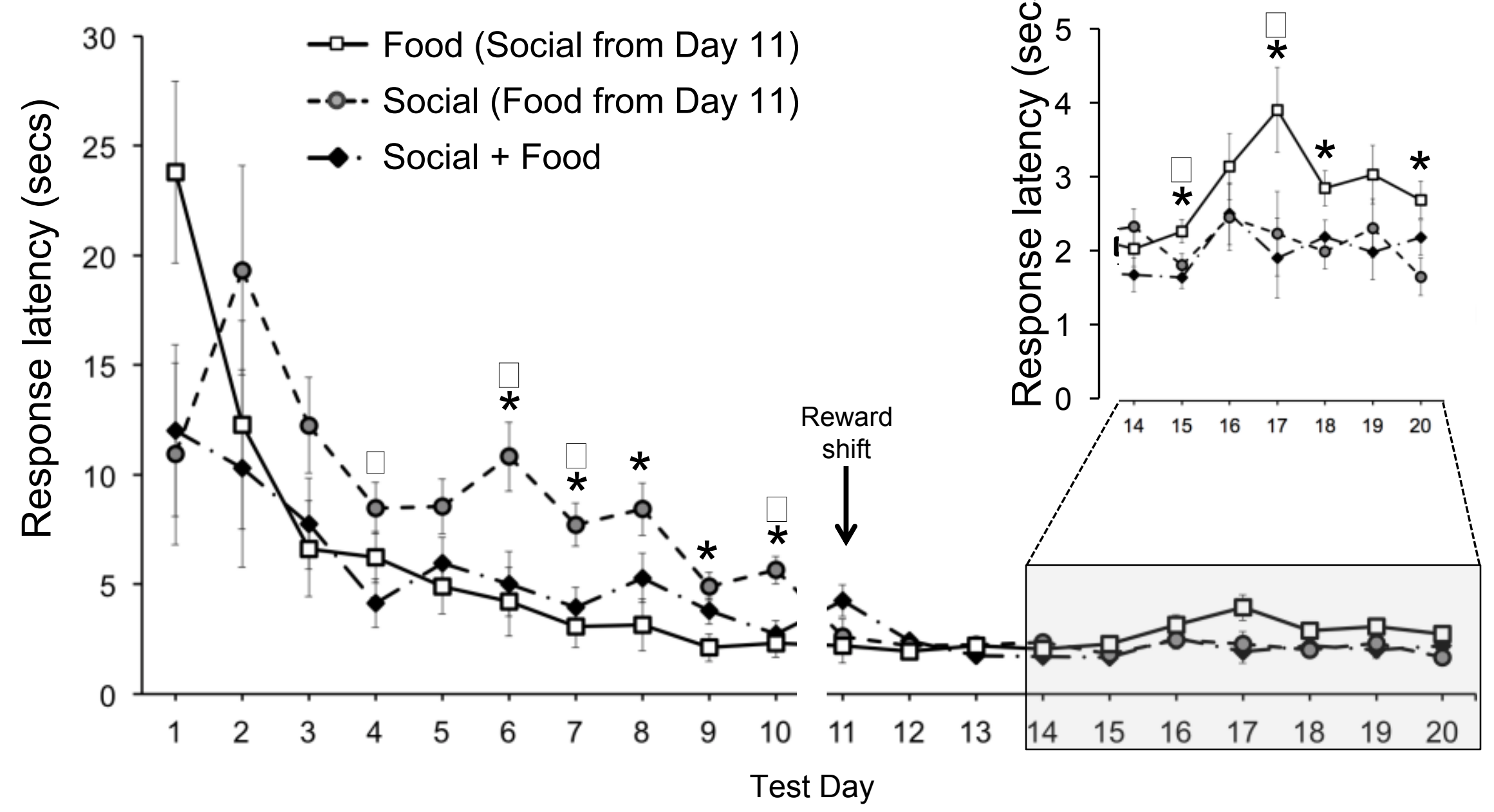




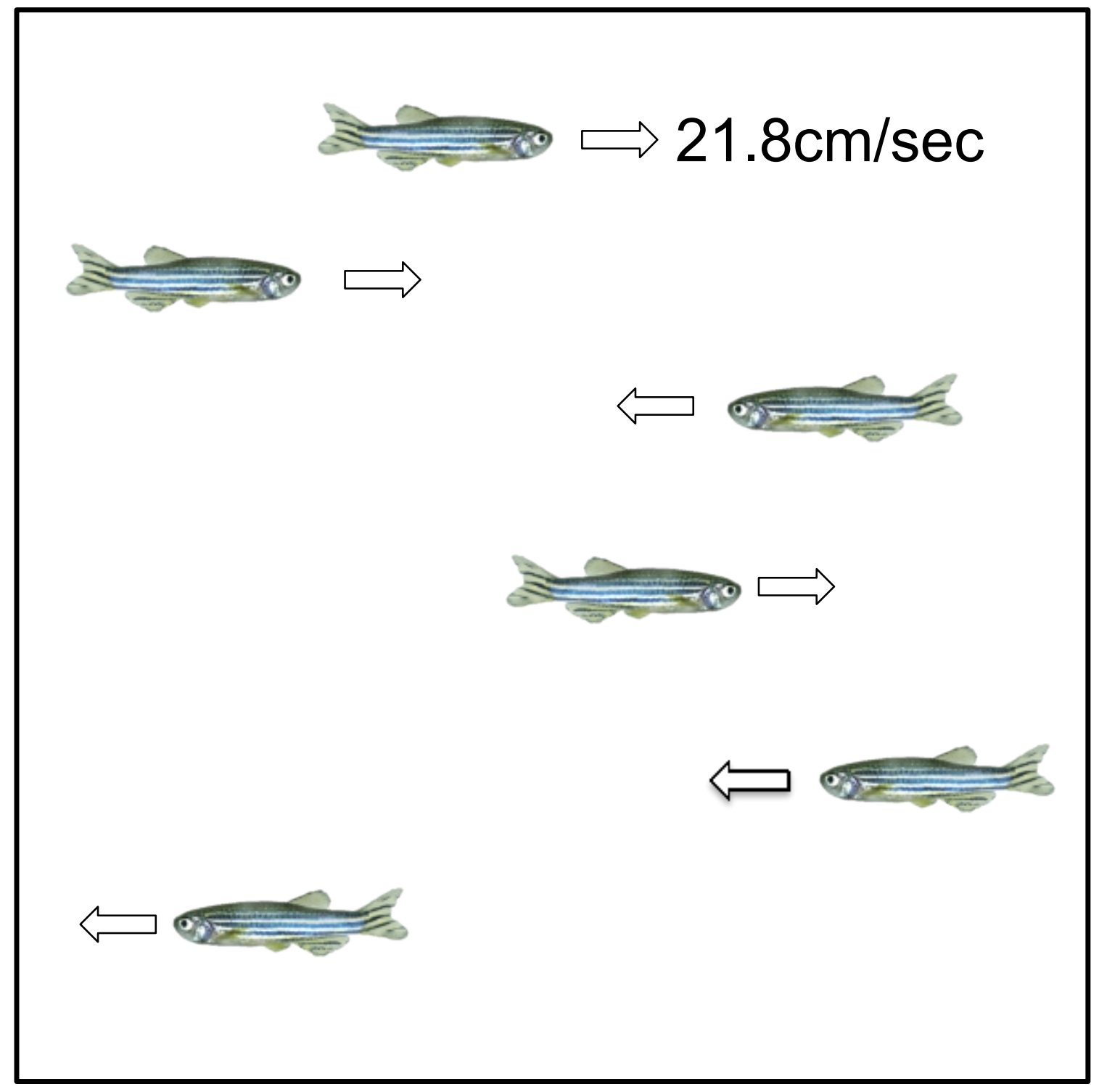

